\title{
A RADIOLOGICAL PROFILE OF FUNGAL SINUSITIS
}

\author{
SURESH PILLAI ${ }^{1}$, AJAY M BHANDARKAR ${ }^{2 *}$, SURAJ S NAIR ${ }^{3}$, ANUSHA TAARINIE JHA ${ }^{4}$, \\ BALAKRISHNAN RAMASWAMY ${ }^{2}$, RASHID AL-ABRI ${ }^{5}$
}

\begin{abstract}
${ }^{1}$ Department of Surgery, ENT Division, Sultan Qaboos University Hospital, Muscat, Oman. ${ }^{2}$ Department of Otorhinolaryngology, Kasturba Medical College, Manipal University, Manipal, Karnataka - 576 104, India. ${ }^{3}$ HCG Cancer Centre, Ahmedabad, Gujarat, India. ${ }^{4}$ Final year MBBS student, Kasturba Medical College, Manipal University, Manipal, Karnataka - 576 104, India. 5Department of Surgery, ENT Division, College of Medicine and Health Sciences, Sultan Qaboos Medical University, Muscat, Oman.
\end{abstract}

Received: 10 May 2017, Revised and Accepted: 05 June 2017

\section{ABSTRACT}

Objectives: To create a radiological profile of fungal sinusitis and determine the radiological differences between fungal and nonfungal sinusitis based on the presence of hyperattenuation, bony erosion, neo-osteogenesis, air-fluid level, and extrasinus extension.

Methods: This is a retrospective, single-blind, case-control study involving the analysis of 119 computed tomography (CT) scans of the paranasal sinuses. Based on the histopathology, they were divided into cases comprising fungal sinusitis and controls of nonfungal sinusitis. Benign and malignant tumors and previously operated cases of fungal sinusitis were excluded from the study. The principal investigators were blinded to the diagnosis. The comparison parameters were hyperattenuation, the presence of air-fluid level, bone erosion, neo-osteogenesis, and extrasinus extension. Data was analyzed by Chi-square and Fischer exact t-test using SPSS 14.0 software and a p $<0.05$ was considered significant.

Results: Our study showed the presence of hyperattenuation, neo-osteogenesis, bone erosion, air-fluid level, extrasinus extension in $75.2 \%, 48.3 \%$, $25.9 \%, 36.2 \%$, and $6.9 \%$ of the cases and $13.1 \%, 16.4 \%, 6.6 \%, 9.8 \%$, and 0 controls, respectively. All the parameters were statistically significant in cases when compared to controls.

Conclusion: Hyperattenuation, neo-osteogenesis, air-fluid level, bone erosion, and extrasinus extension are the parameters on CT imaging that will help routinely assess and differentiate fungal sinusitis from nonfungal sinusitis with considerable accuracy, although, there is an overlap with malignancy when the parameter of bone erosion is considered as a differential diagnosis of chronic invasive fungal sinusitis. It reiterates the fact that history, clinical examination, and laboratory evaluation hold an important role in provisional diagnosis.

Keywords: Fungal sinusitis, Acute invasive fungal sinusitis, Fungus ball, Chronic invasive sinusitis, Allergic fungal sinusitis, Chronic granulomatous sinusitis, Hyperattenuation, Bone erosion, Neo-osteogenesis.

(c) 2017 The Authors. Published by Innovare Academic Sciences Pvt Ltd. This is an open access article under the CC BY license (http://creativecommons. org/licenses/by/4. 0/) DOI: http://dx.doi.org/10.22159/ajpcr.2017.v10i9.19837

\section{INTRODUCTION}

Rhinosinusitis is an inflammatory disorder of the nose and paranasal sinuses that affects $20 \%$ of the human population [1]. The presence of normal fungi in the sinuses has always been a matter of debate. The first case of fungal rhinosinusitis was reported by Plaignaud in 1791 when he detected a "fungal tumor" in a young soldier with maxillary pain. In 1885, Schubert probably first diagnosed a particular variant of fungal rhinosinusitis when he discovered a noninvasive Aspergillus species. Invasive Aspergillosis was first detected by Oppe in 1897 when he detected fungi extending intracranially through the sphenoid sinus [1]. The classification of invasive and noninvasive variants of fungal sinusitis was proposed by Hora in 1965 [2]. McGill reported the fulminant variant of fungal rhinosinusitis in 1980 when he detected rhinocerebral fungal infection [3]. Over the past couple of decades, fungal rhinosinusitis has been increasing, and the reason for this can be attributed to the advances in diagnostic imaging and other adjunctive laboratory investigations. Computed tomography (CT) has been used to differentiate fungal and nonfungal rhinosinusitis based on certain specific features.

\section{Aims and objectives}

To create a radiological profile of fungal sinusitis and to determine the radiological differences between fungal and nonfungal sinusitis on hyperattenuation, the presence of bony erosion, neo-osteogenesis, airfluid level, and extrasinus extension.

\section{MATERIALS AND METHODS}

This is a retrospective, single-blind, case-control study conducted in the department of otolaryngology. Institutional ethical clearance was obtained before the start of this study. 119 CT scans of paranasal sinuses of patients were included in the study, and they were subdivided into two groups. Fungal sinusitis proven by histopathology and fungal culture were taken as cases, and nonfungal sinusitis scans were taken as controls. Cases included patients with allergic fungal rhinosinusitis (AFRS), fungal ball, acute and chronic invasive fungal sinusitis, noninvasive fungal sinusitis, and granulomatous invasive sinusitis. Controls included patients with chronic sinusitis, allergic rhinosinusitis, and chronic granulomatous diseases. Benign and malignant tumors of the nose and paranasal sinuses and previously operated patients of fungal sinusitis were excluded from the study. The comparison parameters were hyperattenuation, the presence of airfluid level, bone erosion, neo-osteogenesis, and extrasinus involvement. The principal investigators were two otorhinolaryngologists with 15 years and 5 years' experience in reading CT scans of the paranasal sinuses and were blinded to the diagnosis. CT scans were reviewed on the parameters described, and a consensus was reached on the result 
of every parameter in every image. Data were analyzed by Chi-square and Fischer exact t-test using SPSS 14.0 software and a $\mathrm{p}<0.05$ was considered significant.

\section{OBSERVATION AND RESULTS}

Our study included 58 patients in the case group with 31 men and 27 women and 61 patients in the control group with 33 men and 28 women. The youngest patient was 7-year-old, and the oldest was 73-year-old. Mean age was 40 years in the case group and 37 years in the control group (Tables 1 and 2).

\section{Hyperattenuation (Fig. 1)}

In our study, the presence of hyperattenuation was noted in $75.9 \%$ of the cases whereas $24.1 \%$ of cases: (AFRS $-13.79 \%$ [ 8 cases], acute invasive sinusitis $-6.89 \%$ [ 4 cases], fungus ball $-1.72 \%$ [ 1 case], chronic invasive sinusitis $-1.72 \%$ [ 1 case]), did not demonstrate the presence of hyperattenuation. However, $13.1 \%$ of the controls also showed the presence of hyperattenuation on CT scan. Although hyperattenuation is a predominant feature of fungal pathology in the sinuses, it was present in nonfungal sinusitis also (13.1\%). However, the presence of hyperattenuation was statistically significant in fungal sinusitis when compared to nonfungal sinusitis $(\mathrm{p}<0.05)$.

\section{Neo-osteogenesis (Fig. 2)}

About $48.3 \%$ of the cases demonstrated the presence of neo-osteogenesis in fungal sinusitis on CT scan against $16.4 \%$ controls. Neo-osteogenesis is a predominant feature of any chronic inflammation of paranasal sinuses, however, in our study, we found that it predominantly occurs in fungal sinusitis when compared to nonfungal sinusitis $(\mathrm{p}<0.05)$.

\section{Bone erosion}

About $25.9 \%$ of the cases and $6.6 \%$ of controls demonstrated the presence of bone erosion. It was interesting to note that among the cases, 6 patients $(10.34 \%)$ had a diagnosis of acute invasive fungal sinusitis (culture - mucor, rhizopus, absidia, and zygomycetes) confirmed by both histopathology and fungal culture whereas 2 cases

Table 1: Parameters as seen in fungal and nonfungal sinusitis

\begin{tabular}{llll}
\hline Findings & $\begin{array}{l}\text { Fungal } \\
\text { sinusitis (\%) }\end{array}$ & $\begin{array}{l}\text { Nonfungal } \\
\text { sinusitis (\%) }\end{array}$ & Significance \\
\hline Hyperattenuation & & & \\
$\quad \begin{array}{l}\text { Present } \\
\text { Absent }\end{array}$ & $44(75.2)$ & $8(13.1)$ & $\mathrm{p}<0.05$ \\
Neo-osteogenesis & $14(24.1)$ & $53(86.9)$ & \\
$\quad \begin{array}{l}\text { Present } \\
\text { Absent }\end{array}$ & $28(48.3)$ & $10(16.4)$ & \\
Bone erosion & $30(51.7)$ & $51(83.6)$ & \\
$\quad \begin{array}{l}\text { Present } \\
\text { Absent }\end{array}$ & $15(25.9)$ & $4(6.6)$ & \\
Air-fluid level & $43(74.1)$ & $57(93.4)$ & \\
$\quad \begin{array}{l}\text { Present } \\
\text { Absent }\end{array}$ & $21(36.2)$ & $6(9.8)$ & \\
$\begin{array}{l}\text { Extrasinus } \\
\text { extension }\end{array}$ & $37(63.8)$ & $55(90.2)$ & \\
$\quad \begin{array}{l}\text { Present } \\
\text { Absent }\end{array}$ & $4(6.9)$ & 0 & \\
\hline
\end{tabular}

(3.44\%) of histopathologically proven AFRS (culture - Aspergillus) and one case $(1.72 \%)$ of histopathologically proven chronic invasive sinusitis (culture - fusarium), demonstrated bone erosion. We had 2 cases $(3.44 \%)$ which showed a diagnostic dilemma where culture demonstrated the presence of (mucor - 1 case, multiple infections with mucor, and rhizopus and Aspergillus - 1 case), but the histopathological diagnosis was AFRS. Pressure-induced bony erosion was the predominant pathogenesis in the 4 cases of nonfungal sinusitis where the lateral wall of the maxillary sinus was the predominant area of erosion. Bone erosion was significant in fungal sinusitis when compared to nonfungal sinusitis, mainly due to the acute invasive sinusitis element in fungal sinusitis ( $\mathrm{p}=0.005)$.

\section{Air-fluid level (Fig. 3)}

About $36.2 \%$ of cases and $9.8 \%$ controls demonstrated the presence of air-fluid level on CT scan. The air-fluid level in the scans, (chronic

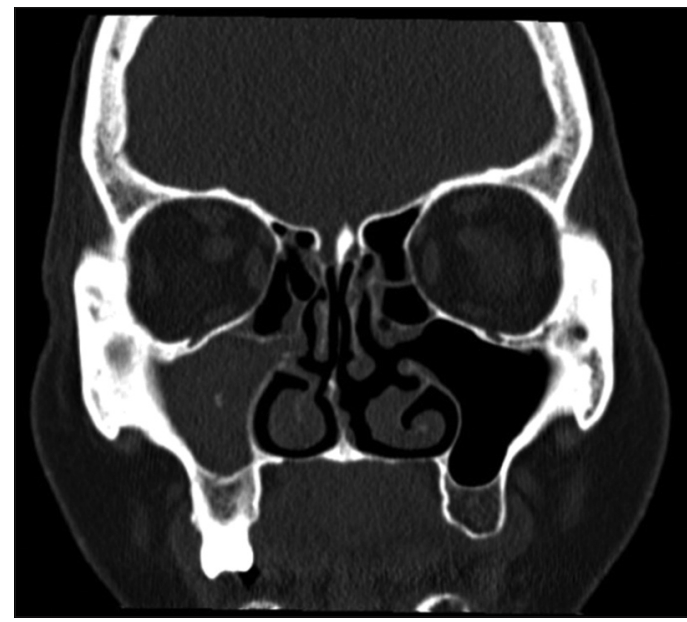

Fig. 1: Hyperattenuation in the right maxillary sinus

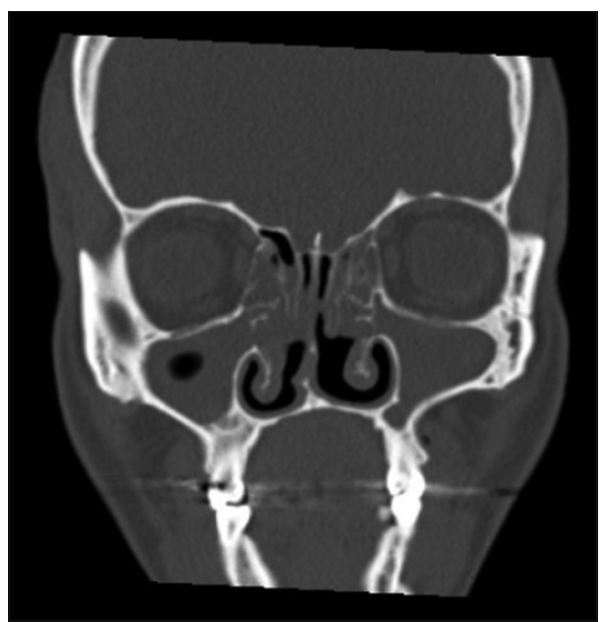

Fig. 2: Neo-osteogenesis of the maxillary sinus wall

Table 2: Parameters in the various subtypes of fungal sinusitis

\begin{tabular}{|c|c|c|c|c|c|}
\hline Subtypes of Fungal Sinusitis & Hyperattenuation (\%) & Neo-osteogenesis (\%) & $\begin{array}{l}\text { Bone } \\
\text { erosion (\%) }\end{array}$ & $\begin{array}{l}\text { Air-fluid } \\
\text { level (\%) }\end{array}$ & $\begin{array}{l}\text { Extrasinus } \\
\text { extension (\%) }\end{array}$ \\
\hline Acute invasive fungal sinusitis & $4(57.14)$ & $1(14.28)$ & $5(71.42)$ & Nil & $2(28.57)$ \\
\hline Chronic invasive fungal sinusitis & $1(25)$ & $2(50)$ & $1(25)$ & $3(75)$ & $1(25)$ \\
\hline $\begin{array}{l}\text { Chronic granulomatous invasive } \\
\text { sinusitis }\end{array}$ & Nil & $\mathrm{Nil}$ & Nil & Nil & Nil \\
\hline Fungus ball & $1(33.33)$ & $1(33.33)$ & Nil & $1(33.33)$ & Nil \\
\hline Allergic fungal sinusitis & $8(18.18)$ & $24(54.54)$ & $4(9.09)$ & $17(38.63)$ & Nil \\
\hline
\end{tabular}


invasive sinusitis - 5.17\% [ 3 cases], fungus ball - 1.72\% [ 1 case], and AFRS - 29.31\% [17 cases]), was mainly due to the presence of an acute sinus infection within the predominant fungal sinusitis. The presence of air-fluid level in both fungal and nonfungal sinusitis can be attributed to acute inflammation within the sinuses at the time of presentation.

\section{Extrasinus extension (Fig. 4)}

About $6.9 \%$ cases (acute invasive sinusitis - 5.17\% [3 cases], chronic invasive sinusitis - $1.72 \%$ [ 1 case]) demonstrated the presence of extrasinus extension against no extrasinus extension in nonfungal sinusitis on CT scan. The extrasinus extension is a significant feature of invasive fungal sinusitis and was statistically significant when compared to nonfungal sinusitis $(\mathrm{p}<0.05)$.

\section{DISCUSSION}

Upper and lower airways are frequently colonized by various microorganisms including fungi due to inhalation of spores from the external environment. Aspergillus species are the most common colonizers in the paranasal sinuses. Lack of host immune responsiveness is eventually responsible for the symptomatic manifestation of fungal disease [4]. Fungal sinusitis has been classified into invasive and noninvasive fungal rhinosinusitis. Invasive fungal rhinosinusitis has been further subclassified into an acute fulminant form, granulomatous form and a chronic invasive form. Noninvasive fungal rhinosinusitis has been subclassified into AFRS, fungal mycetoma, and saprophytic variant [5].

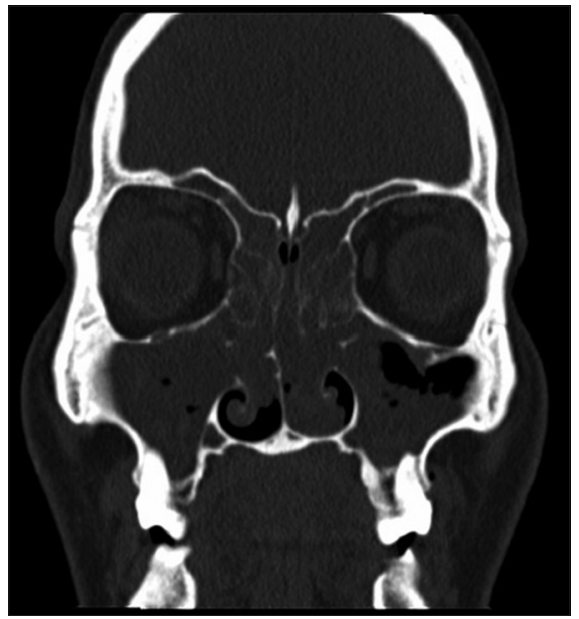

Fig. 3: Air-Fluid level in the left maxillary sinus

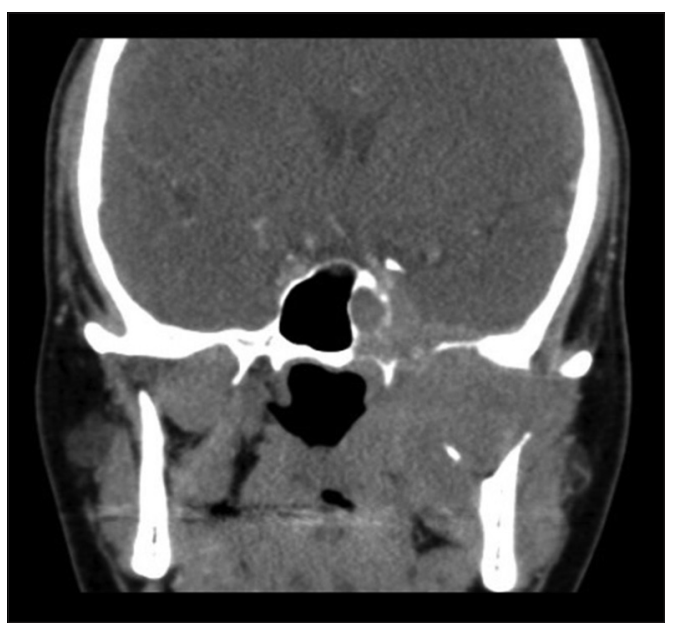

Fig. 4: Extrasinus extension of fungal sinusitis from the left sphenoid sinus
Acute invasive fungal rhinosinusitis

Imaging in acute fulminant invasive fungal rhinosinusitis (AFIFRS), is essential to determine the bony and soft tissue involvement of the aggressive fungus involved in this debilitating condition. Magnetic resonance imaging (MRI) is extremely sensitive and has a high negative predictive value for the early changes in AFIFRS [4]. However, the specificity and negative predictive value of MRI is similar to CT scan [6]. The pathogenic spread of AFIFRS starts in the mucosa of the middle turbinate eventually spreading to the paranasal sinuses. Intracranial or extrasinus soft tissue involvement is either through sinus wall bone erosion or hematogenous spread in case of an intact bony wall which may lead to life-threatening complications such as internal carotid artery invasion, cavernous sinus thrombosis, intracranial abscesses, infarct, and hemorrhage [4,7]. The typical findings of AFIFRS on CT scan as determined by literature are (1) opacification of involved sinuses (2) focal bony erosion (3) sinus and lateral nasal wall soft tissue thickening (4) premaxillary fat pad infiltration due to vascular infiltration (5) hyperattenuation due to the presence of fungal secretions and (6) heterogenicity due to the presence of fungal hyphae, trace metals, depleted water or elevated protein content or a combination of all $[4,7-9]$ The early features on CT scan are consistent with any form of sinus inflammation as it shows nasal soft tissue thickening which is not specific to AFIFRS, however, late features of retroantral infiltration of the fat pad, extrasinus and intracranial involvement are more specific for AFIFRS [4,10]. DelGaudio et al [11]. in their study of 23 patients of AFIFRS showed sinus soft tissue thickening $(91 \%)$, opacification $(91 \%)$, bony erosion $(35 \%)$, orbit involvement $(26 \%)$, facial soft tissue thickening (22\%), and air-fluid level $(0.08 \%)$ of their patients with a statistically significant soft tissue thickening $(p<0.001)$, and nonsignificant bony erosion $(p>0.05)$ when compared to the control group. In our study, seven patients were proven to have AFIFRS on histopathological analysis and imaging. 57.14\% showed hyperattenuation, $71.42 \%$ showed bony erosion, $28.57 \%$ showed extrasinus extension, $14.28 \%$ showed neo-osteogenesis, and none showed air-fluid levels on imaging.

Chronic invasive fungal rhinosinusitis and chronic granulomatous fungal rhinosinusitis

The radiological distinction between chronic invasive and chronic granulomatous fungal rhinosinusitis is difficult. However, it is possible to differentiate from chronic rhinosinusitis. Both these chronic invasive fungal infections invade the epithelium, subepithelial tissue, blood vessels, and sinus walls [4]. The commonly involved sinuses are the anterior ethmoid and sphenoid sinuses. The radiological findings are similar to AFIFRS, but the duration of these variants are in months. The classical radiological findings of chronic fungal rhinosinusitis are: (1) Unilateral sinus involvement, (2) homogeneity in contrast enhancement, (3) bone erosion, (4) hyperattenuation with the destruction of bony sinus walls, and (5) extrasinus involvement is extremely higher when compared to intrasinus involvement $[4,7,8,12,13]$. The complications of chronic fungal invasive infections are similar to AFIFRS, and the duration might prompt the clinician to consider a differential diagnosis of malignancy. Reddy et al [14]. studied 17 cases of chronic invasive fungal sinusitis which were differentiated on histopathology into 15 cases of granulomatous invasive fungal sinusitis and 2 cases of chronic invasive fungal sinusitis, and they demonstrated homogeneity with no focal hyperattenuation and localized bony erosion at the site of the extrasinus expansion of the fungal lesion. They also observed sinus expansion and moderately intense homogenous contrast enhancement of the fungal lesion. In our study, we noted 4 cases of chronic invasive fungal sinusitis without any granulomatous invasive fungal sinusitis. $25 \%$ showed hyperattenuation, $50 \%$ showed neo-osteogenesis, $25 \%$ showed bony erosion, $75 \%$ showed air-fluid level, and $25 \%$ showed extrasinus extension.

\section{Fungus ball (mycetoma)}

This is a noninvasive variant of fungal sinusitis which characteristically involves only a single paranasal sinus.[4] Apart from solitary involvement, the other characteristic features of mycetoma on CT 
imaging are: (1) Dense spot in the core of the lesion with surrounding bony sclerosis, (2) punctate calcifications within the mycetoma, and (3) hyperattenuating mucosal thickening circumferentially around the core. The fungus ball takes the contour of the sinus lumen $[4,7,8,15]$. Chen and Ho[16] studied 96 cases of mycetoma of the paranasal sinuses in which $27.1 \%$ showed heterogeneous density, $97.1 \%$ showed absent air-fluid levels, $72.9 \%$ showed sinus wall erosion and peripheral bony sclerosis, $70.8 \%$ showed calcification, and $50 \%$ showed mucosal thickening. In our study, we had 3 cases of fungus ball. $33.3 \%$ showed hyperattenuation, $33.3 \%$ showed air-fluid level, $33.3 \%$ showed neoosteogenesis, and none showed bony erosion or extrasinus extension.

\section{Allergic fungal rhinosinusitis}

Bent and Kuhn laid down the criteria which combine the clinical, laboratory and radiological findings to diagnose AFRS [17]. AFRS usually demonstrates a pansinusitis with ethmoid sinus being the most common paranasal sinus to be involved [4]. CT imaging of AFRS demonstrates the following pathognomonic findings: (1) Hyperattenuation due to the presence of allergic mucin, (2) expansion of bony sinus walls, (3) remodeling of bony sinus wall, (4) lack of enhancement on contrast CT, (5) smooth bony erosion, and (6) double density sign with a starry sky or serpiginous appearance $[4,7,8,15,18]$. As per literature, $20 \%$ of all AFRS demonstrate ethmoid bone erosion on CT imaging with associated sinus expansion [19]. Our study had 44 cases of diagnosed AFRS. $18.18 \%$ showed hyperattenuation, $54.54 \%$ showed hyperostosis, $9.09 \%$ showed bony erosion, $38.63 \%$ showed airfluid level, and none showed extrasinus extension.

Our study is limited by the fact that the outcome measures were subjectively assessed even though it was by well-qualified otolaryngologists. It gave an insight into the various parameters that can be assessed by CT scan with accuracy to differentiate fungal sinusitis from nonfungal sinusitis. Although a few parameters on CT imaging are telltale diagnostic of fungal sinusitis, it is only complementary to history, clinical examination, and laboratory investigations to confirm the subtype of fungal sinusitis. Literature also shows evidence that endophytic fungi can be used in the generation of anticancer agents [20]. CT imaging alone will not be sufficient to accurately diagnose a subtype of fungal sinusitis although it helps us to differentiate it from nonfungal sinusitis. Treatment of fungal rhinosinusitis whether topical or systemic is based on the accuracy of various investigations [21].

\section{CONCLUSION}

The diagnosis of fungal rhinosinusitis by CT imaging is challenging. Hyperattenuation, neo-osteogenesis, air-fluid level, bone erosion, and extrasinus extension are the parameters that will help routinely assess and differentiate fungal sinusitis from nonfungal sinusitis with considerable accuracy, although, there is an overlap with malignancy when the parameter of bone erosion is considered as a differential diagnosis of chronic invasive fungal sinusitis. It reiterates the fact that history, clinical examination, and laboratory evaluation hold a key role in provisional diagnosis. Advances in imaging form a valuable adjunct tool for diagnosis of paranasal sinus pathologies.

\section{REFERENCES}

1. Chakrabarti A, Das A, Panda NK. Overview of fungal rhinosinusitis. Indian J Otolaryngol Head Neck Surg 2004;56(4):251-8.

2. Hora JF. Primary aspergillosis of the paranasal sinuses and associated areas. Laryngoscope 1965;75:768-73.

3. McGill TJ, Simpson G, Healey GB. Fulminant Aspergillosis of the nose and paranasal sinuses: A new clinical entity. Laryngoscope 1980;90:748-54

4. Gorovoy IR, Kazanjian M, Kersten RC, Kim HJ, Vagefi MR. Fungal rhinosinusitis and imaging modalities. Saudi J Ophthalmol 2012;26(4):419-26.

5. deShazo RD, Chapin K, Swain RE. Fungal sinusitis. N Engl J Med 1997;337(4):254-9.

6. Groppo ER, El-Sayed IH, Aiken AH, Glastonbury CM. Computed tomography and magnetic resonance imaging characteristics of acute invasive fungal sinusitis. Arch Otolaryngol Head Neck Surg 2011;137(10):1005-10

7. Aribandi M, McCoy VA, Bazan $\mathrm{C} 3^{\text {rd }}$. Imaging features of invasive and noninvasive fungal sinusitis: A review. Radiographics 2007;27(5):1283-96.

8. Mossa-Basha M, Ilica AT, Maluf F, Karakoç Ö, Izbudak I, Aygün N. The many faces of fungal disease of the paranasal sinuses: CT and MRI findings. Diagn Interv Radiol 2013;19(3):195-200.

9. Momeni AK, Roberts CC, Chew FS. Imaging of chronic and exotic sinonasal disease: Review. AJR Am J Roentgenol 2007;189 6 Suppl:S35-45.

10. Silverman CS, Mancuso AA. Periantral soft-tissue infiltration and its relevance to the early detection of invasive fungal sinusitis: $\mathrm{CT}$ and MR findings. AJNR Am J Neuroradiol 1998;19(2):321-5.

11. DelGaudio JM, Swain RE Jr, Kingdom TT, Muller S, Hudgins PA. Computed tomographic findings in patients with invasive fungal sinusitis. Arch Otolaryngol Head Neck Surg 2003;129(2):236-40.

12. Dahniya MH, Makkar R, Grexa E, Cherian J, Al-Marzouk N, Mattar M, et al. Appearances of paranasal fungal sinusitis on computed tomography. Br J Radiol 1998;71(843):340-4.

13. Stringer SP, Ryan MW. Chronic invasive fungal rhinosinusitis. Otolaryngol Clin North Am 2000;33(2):375-87.

14. Reddy CE, Gupta AK, Singh P, Mann SB. Imaging of granulomatous and chronic invasive fungal sinusitis: Comparison with allergic fungal sinusitis. Otolaryngol Head Neck Surg 2010;143(2):294-300.

15. Khattar VS, Hathiram BT. Radiologic appearances in fungal rhinosinusitis. Otorhinolaryngol Clin Int J 2009;1(1):15-23.

16. Chen JC, Ho CY. The significance of computed tomographic findings in the diagnosis of fungus ball in the paranasal sinuses. Am J Rhinol Allergy 2012;26(2):117-9.

17. Bent JP $3^{\text {rd }}$, Kuhn FA. Diagnosis of allergic fungal sinusitis. Otolaryngol Head Neck Surg 1994;111(5):580-8.

18. Sari-Aslani F, Khademi B, Vatanibaf MR, Noroozi MS. Diagnosis of allergic fungal rhinosinusitis. Iran J Med Sci 2006;31(4):200-3.

19. Nussenbaum B, Marple BF, Schwade ND. Characteristics of bony erosion in allergic fungal rhinosinusitis. Otolaryngol Head Neck Surg 2001;124(2):150-4.

20. Firodiya AD, Tenguria RK. Endophytic fungi: Treasure for anticancerous compounds. Int J Pharm Pharm Sci 2016;8(8):35-42.

21. Mirmoezzi MS, Yazdi MS, Gholami O. Comparative study on the efficacy of mometasone and fluticasone nasal sprays for treatment of allergic rhinitis. Int J Pharm Pharm Sci 2017;9(3):211-4. 